\title{
Sarcopenia em portadores de doença renal crônica em diálise peritoneal no Sistema Único de Saúde
}

\author{
"Sarcopenia in individuals with chronic renal failure in peritoneal \\ dialysis in the Unified Health System"
}

\author{
Sheila Borges' • Renata Costa Fortes ${ }^{2}$
}

\begin{abstract}
RESUMO
Objetivo:Avaliar a sarcopenia e fatores associados em portadores de doença renal crônica de um programa de diálise peritoneal vinculado ao Sistema Único de Saúde do Distrito Federal. Metodologia: Estudo transversal analítico, com indivíduos de ambos os sexos, acima de 18 anos de idade, com mais de três meses em diálise. As variáveis avaliadas foram: tempo de diálise, medidas antropométricas; presença de comorbidades; níveis séricos de albumina, creatinina, ureia, ferritina, ingestão de proteína e calorias. Houve a aplicação do instrumento SARC-F para triagem da sarcopenia.A avaliação da massa muscular foi realizada por meio de bioimpedância por espectroscopia, além da mensuração da força de preensão palmar, performance física.A presença de desnutrição foi determinada pela avaliação subjetiva global de sete pontos (ASG-7p). O nível de significância

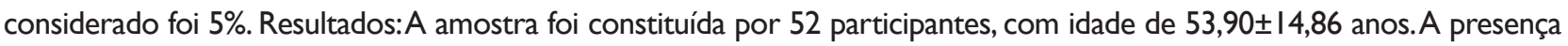
da sarcopenia esteve em 15 (28,85\%) participantes. Essa condição foi predominante em homens e hipertensos, observou-se diferença estatística entre a sarcopenia em relação à ASG-7p $(p=0,043)$, ângulo de fase $(p=0,005)$ e albumina $(p<0,00$ I $)$. Níveis reduzidos de albumina aumentaram as chances da ocorrência de sarcopenia $(R R, 22,22 ; 95 \% I C \quad 0,00-0,80$; $p=0,034)$. Conclusão:A sarcopenia é prevalente na doença renal e a albumina é um fator independente para essa condição.

Palavras-chave: Diálise Peritoneal; Insuficiência Renal, Sarcopenia.
\end{abstract}

\begin{abstract}
Objective:To evaluate sarcopenia and associated factors in individuals with chronic renal failure in a peritoneal dialysis program of the Unifed Health System in the Federal District. Method: Cross-sectional analytical study, individuals both sexes, over 18 years of age, and more than three months in dialysis. The variables evaluated were: dialysis time, anthropometric measurements; presence of comorbidities; serum levels of albumin, creatinine, urea, ferritin, protein and calorie intake. The SARC-F instrument was applied to screening for sarcopenia. The evaluation of muscle mass was perfomed by means of bioimpedance by spectroscopy, in addition to the measurement of handgrip strength, physical performance. Malnutrition was determined by a seven-point subjective global assessment (SGA-7p). The level of significance considered was $5 \%$. Results: The sample was created by 52 participants, aged $53,90 \pm$ I4,86 years. The presence of sarcopenia was $15(28,85 \%)$ individuals. This condition was predominant in males and hypertensive individuals, with a statistical difference between the sarcopenia in relation to GSA-7p $(p=0,043)$, phase angle $(p=0,005)$ and albumin $(p<0,00 \mathrm{I})$. Reduced album levels increased the times of sarcopenia (RR, $22,22 ; 95 \% \mathrm{Cl} 0,00-0,80 ; p=0,034)$. Conclusion: Sarcopenia is prevalente in chronic renal failure and albumin is an independente fator for this condition.

Keywords: Peritoneal Dialysis; Renal Insufficiency; Sarcopenia.
\end{abstract}

I Nutricionista, Mestre em Ciências para a Saúde, Escola Superior de Ciências da Saúde, Fundação de Ensino e Pesquisa em Ciências da Saúde. Brasília, Distrito Federal. E-mail: sbnutril2@hotmail.com. ORCID: https://orcid.org/0000-0002-5407-042I

2 Nutricionista, Doutora em Nutrição Humana pela Universidade de Brasília. Docente do Mestrado Profissional em Ciências para a Saúde da Escola Superior de Ciências da Saúde, Fundação de Ensino e Pesquisa em Ciências da Saúde. Brasília, Distrito Federal. E-mail: fortes.rc@gmail.com. ORCID: https://orcid.org/00000002-0583-645I

Autor correspondente: Sheila Borges. E-mail: sbnutril2@hotmail.com. 


\section{INTRODUÇÃO}

A doença renal crônica (DRC) é um importante problema de saúde pública e apresenta elevada morbidade e mortalidade. É uma enfermidade que consiste na lesão renal e/ou perda lenta, progressiva, irreversível, da função dos rins. Segundo o estudo "Global Burden of Disease Study $2017^{\prime \prime(1), ~ u m a ~ a n a ́ l i s e ~ s i s t e m a ́ t i c a ~ s o b r e ~} 282$ causas de mortes em 195 países entre os anos de 1980 e 2017, a DRC configurou-se como a $16^{\text {a }}$ causa de óbitos.

Recentemente, a Sociedade Brasileira de Nefrologia divulgou uma comparação do perfil clínico dos pacientes em diálise crônica no Brasil entre os anos de 2009 e 2018, segundo os resultados dessa análise, observou-se um aumento médio anual de 5.587 pacientes nesse período, correspondente a $6,4 \%$ ao ano(2).

O tratamento da DRC consiste em terapia de substituição renal por meio das seguintes modalidades: diálise peritoneal (DP), hemodiálise (HD) e o transplante. A DP pode ser realizada por três tipos: diálise peritoneal ambulatorial contínua (DPAC), diálise peritoneal automatizada (DPA) e diálise peritoneal intermitente (DPI) ${ }^{(3)}$.

No Brasil, há um predomínio da indicação da HD em relação à $\mathrm{DP}$, sendo apenas $6,9 \%$ dos pacientes nessa última modalidade ${ }^{(2)}$. Na DP, retira-se as escórias e o excesso de líquidos através da membrana peritoneal. É uma modalidade que vem sendo apontada como uma opção que permite maior autonomia e flexibilidade ao paciente na realização do tratamento, possibilita manutenção das atividades diárias, melhora na qualidade de vida, em especial, no que se refere à dimensão social, minimizando assim o impacto da doença( ${ }^{(3)}$.

Dos pacientes em DP, a maioria realiza DPA, procedimento por meio de uma cicladora, podendo a diálise ser feita em domicílio por pessoal previamente treinado e não exige acesso vascular, uma vez que, o cateter utilizado é implantado por procedimento cirúrgico na cavidade peritoneal ${ }^{(3)}$.

Os portadores de DRC carregam consigo um enorme risco de mortalidade precoce, especialmente por doenças cardiovasculares. A patogênese da doença cardiovascular nessa população é complexa e, além dos fatores de risco tradicionais como a presença de hipertensão arterial, diabetes e dislipidemias, há outros fatores emergentes como: anemia, distúrbio mineral ósseo, exacerbação de estresse oxidativo e, possivelmente, presença de sarcopenia ${ }^{(4)}$.

A sarcopenia é uma progressiva e generalizada desordem do músculo esquelético que envolve a perda acelerada de massa e função muscular associada a eventos adversos aumentados como quedas, declínio da funcionalidade, hospitalizações recorrentes e um prognóstico negativo ${ }^{(5)}$. É uma condição encontrada em diversos estágios da doença renal, sendo um processo multifatorial que inclui a inatividade física, remodelamento de unidades motoras e redução da síntese de proteínas.
As situações que contribuem para a manifestação da sarcopenia são: alterações hormonais, grau de inflamação crônica, repouso prolongado no leito, além de uma dieta abaixo das recomendações ${ }^{(4)}$. Com isso, a investigação dessa condição na DRC é de suma importância na assistência nutricional e para prevenção de desfechos negativos.

Dessa forma, o objetivo desse estudo foi avaliar a sarcopenia e fatores associados em portadores de doença renal crônica de um programa de diálise peritoneal vinculado ao Sistema Único de Saúde do Distrito Federal.

\section{MÉTODOS}

\section{Delineamento do estudo}

Tratou-se de um estudo transversal analítico, realizado com portadores de DRC em DPA acompanhados na unidade de nefrologia do Hospital Regional de Taguatinga, da Secretaria de Estado de Saúde do Distrito Federal (SES-DF) entre os meses de maio a agosto de 2019.

\section{Casuística}

A amostra foi obtida por conveniência, sendo que os participantes incluídos tinham acima de 18 anos de idade, ambos os sexos, com taxa de filtração glomerular (TFG) menor que $15 \mathrm{~mL} /$ minuto $/ 1,73 \mathrm{~m}^{2}$, em realização do tratamento dialítico por mais de três meses. Foram excluídos os portadores de DRC que, durante o momento da coleta dos dados, estavam institucionalizados e/ou hospitalizados, os indivíduos portadores de síndrome de imunodeficiência e câncer (síndromes consumptivas), gestantes, acamados, amputados, ou os que não conseguiram realizar o teste de desempenho físico para avaliação da sarcopenia.

\section{Caracterização da amostra}

As informações sobre idade, sexo, tempo de diálise em meses, causas da DRC e presença de hipertensão e diabetes foram obtidas em entrevista no momento das consultas mensais de rotina ou por meio de informações obtidas nos prontuários, registrados em questionário previamente elaborado.

\section{Antropometria}

As medidas antropométricas como peso, estatura, circunferência braquial (CB), dobra cutânea triciptal (DCT), circunferência abdominal (CA) e circunferência da panturrilha (CP) foram obtidas sem a presença de líquido na cavidade abdominal do participante para evitar a influência de retenção hídrica. Todas as medidas foram realizadas pela própria pesquisadora, conforme as técnicas estabelecidas ${ }^{(6)}$.

Para aferição do peso atual foi utilizada uma balança digital portátil, marca Camry®.A estatura foi determinada pelo estadiômetro portátil, marca Sanny®. Os participantes foram orientados a ficarem descalços e centrali- 
zados, eretos, braços soltos ao longo do corpo e olhar na linha do horizonte, conforme plano de Frankfurt ${ }^{(6)}$. Após a obtenção do peso e da estatura foi calculado o índice de massa corporal (IMC) por meio da razão entre o peso corporal e a estatura ao quadrado $\left(\mathrm{kg} / \mathrm{m}^{2}\right)$.

A mensuração da CB foi realizada com o auxílio de uma fita métrica inextensível com o braço do participante flexionado em direção ao tórax, formando um ângulo de $90^{\circ}$, no ponto médio entre o acrômio e o olécrano da ulna, preferencialmente, no braço relaxado não dominante com a palma da mão voltada para baixo. Contornou-se o braço com a fita no ponto marcado de forma ajustada evitando compressão da pele ou folga ${ }^{(6)}$.

A aferição da DCT foi realizada por meio de adipômetro marca Lange $\AA$. Utilizou-se o braço relaxado e estendido não dominante com o participante na posição vertical. A princípio, identificou-se o ponto médio entre o acrômio e o olécrano, na posição posterior, com o braço flexionado, formando um ângulo de $90^{\circ}$. O adipômetro foi posicionado perpendicularmente à dobra, exatamente no local marcado. Suavemente pinçou-se a pele no ponto marcado entre as extremidades do adipômetro, enquanto se manteve a dobra cutânea tracionada. A leitura foi feita com aproximação de um milímetro, dois a três segundos após o alinhamento do ponteiro. Essa aferição foi feita por três vezes, calculando-se a média aritmética dos valores obtidos( ${ }^{(6)}$.

A medida da CA foi determinada, por meio da fita métrica, ao nível da maior extensão abdominal, nem sempre sendo ao nível da cicatriz umbilical( ${ }^{(6)}$. A aferição da CP obteve-se com o participante sentado, com os joeIhos flexionados a $90^{\circ}$, com o calcanhar apoiado sobre uma cadeira. Moveu-se a fita métrica na panturrilha, para cima e para baixo até encontrar o perímetro máximo, ou seja, a maior circunferência ${ }^{(6)}$.

Para cálculo da circunferência muscular do braço $(C M B)$ foi utilizada a fórmula: $C M B(\mathrm{~cm})=C B(\mathrm{~cm})-$ $\{D C T$ em milímetros $\times 0,3 \mid 4\}$. Por haver diferenças entre os valores de referência para homens e mulheres, obtevese valores de adequação percentual para CMB.Tais valores foram obtidos pela razão entre o valor da medida realizada e o valor de referência da medida (percentil 50). Os resultados foram classificados segundo Frisancho ${ }^{(7)}$.

\section{Avaliação subjetiva global de sete pontos (ASG-7p)}

Esse método integrativo contém seis domínios: mudança involuntária de peso corporal nos últimos seis meses, ingestão alimentar nas últimas duas semanas, sintomas gastrointestinais persistentes por mais de duas semanas, capacidade funcional reduzida relacionada à nutrição, estado de doenças relacionadas às necessidades nutricionais, perda de massa muscular visível em pelo menos três áreas, perda de tecido adiposo visível em pelo menos três áreas e presença de edema relacionado à subnutrição(8).
Cada domínio foi pontuado, de acordo com a intensidade da alteração encontrada, variando de um a sete pontos. A pontuação mais frequente entre todos os domínios avaliados representou a nota final e, a partir desta, os participantes foram classificados quanto ao estado nutricional. Indivíduos com predominância de pontuação um ou dois foram classificados como desnutridos graves; os com pontuação entre três a cinco, como leve ou moderadamente desnutridos; e os com pontuação igual ou acima de seis receberam a classificação de bem nutridos ou com risco muito leve para desnutrição( ${ }^{(8)}$.

\section{Avaliação da sarcopenia}

Considerou-se os passos propostos pelo EWGSOP(9). Inicialmente, o instrumento do SARC-F foi aplicado para triagem da sarcopenia. Participantes com resultado de zero a três pontos não possuíam risco para sarcopenia, já o resultado acima de quatro pontos sugeriu presença de sarcopenia ${ }^{(10)}$.

A análise da composição corporal foi realizada por meio de BIS utilizando o aparelho Body Composition Monitor (BCM) marca Fresenius Medical Care $囚$, Bad Homburg,Alemanha, tetrapolar, multifrequencial. A BIS foi feita no mesmo momento de mensuração das medidas antropométricas.

O participante foi posicionado em decúbito dorsal horizontal com braços e pernas afastados do tronco, aproximadamente, $30^{\circ}$. Os participantes foram orientados a não realizar exercício oito horas antes, não consumir álcool nas 12 horas anteriores, não consumir nenhum tipo de alimento ou bebida por pelo menos quatro horas antecedentes, esvaziar a bexiga 30 minutos antes do teste, não passar nenhuma espécie de loção hidratante no corpo e retirar objetos de metal (celular, chaves, cintos), inclusive os presos ao corpo como brincos, anéis e relógios. Os eletrodos foram posicionados no lado não dominante, na região dorsal na mão (um entre a cabeça da ulna e o rádio, e o outro na falange proximal do terceiro dedo) e no pé (um eletrodo entre os maléolos medial e lateral e outro na região do terceiro metatarso). Nesses locais a pele foi limpada com álcool $70 \%$ e algodão.

O parâmetro de massa muscular obtido na avalição pela BIS foi o índice de tecido magro (LTI), resultado da razão da massa magra do corpo em $\mathrm{kg}$ pela altura em metros ao quadrado. $O$ valor reduzido de LTI foi definido pelo resultado abaixo do percentil 10 , segundo o sexo e a idade, da referência de National Health and Nutrition Examination Survey (NHANES) 2003-2004(11). Os valores de referência para LTI foram definidos entre os percentis 10 e 90 , segundo idade e sexo.

A confirmação da presença de sarcopenia foi determinada pela combinação de redução da LTI e da força de preensão palmar (FPP). Esse último parâmetro foi mensurado pelo lado dominante utilizando dinamômetro hi- 
dráulico Jamar®, marca Patterson Medical, Reino Unido, escala de 0 a $90 \mathrm{~kg}$, uma precisão de $1,0 \mathrm{~kg}$. Os participantes estavam sentados em uma cadeira adequada para estatura no intuito de garantir que os seus braços pudessem descansar confortavelmente. Os mesmos foram instruídos a autoajustar o dinamômetro para encaixe confortável ao tamanho da mão com o objetivo de obter o melhor desempenho. Com o cotovelo dobrado em um ângulo de $90^{\circ}$, antebraço e punho em uma posição neutra, os participantes foram instruídos a aplicar o máximo de força possível ao aparelho. Três medidas foram realizadas com um descanso de um minuto entre elas. Considerou-se o maior valor de FPP obtido. Os pontos de corte para adultos considerados, para classificação da redução da FPP, foram de acordo com Massy-WestropP et al. ${ }^{(12)}$, segundo variação conforme o sexo e a faixa etária. Já para idosos, considerou-se redução da FPP pelos pontos de corte propostos pelo EWGSOP(9), sendo para homens $<27 \mathrm{~kg}$ e mulheres $<16 \mathrm{~kg}$.

O teste de velocidade de marcha foi realizado para confirmação da sarcopenia severa. $O$ participante caminhou uma distância de quatro metros, previamente demarcada por fitas fixadas ao chão, com tempo mensurado por meio de cronômetro digital. Considerou-se uma baixa velocidade de marcha valores maiores que 0,8 metros por segundo, o que corresponde à realização do percurso de quatro metros em um tempo superior a 5 segundos $^{(9)}$.

$O$ valor do ângulo de fase, também sugerido como indicador nutricional, foi obtido pela avaliação da composição corporal por meio do aparelho do BCM foi obtido na frequência de $50 \mathrm{kHz}$. É o resultado da derivação utilizando a relação geométrica entre as medidas de resistência $(\mathrm{R})$ e a reactância $(\mathrm{Xc})$, ambas variáveis fornecidas pela bioimpedância, podendo ser calculado por meio da fórmula:Arco-tangente $\left(X_{c} / R\right) \times 180^{\circ} / 3,14^{(13)}$.

\section{Parâmetros laboratoriais}

Os resultados dos exames laboratoriais foram obtidos nos prontuários dos participantes, sendo as análises realizadas mensalmente no próprio laboratório do hospital. Os parâmetros bioquímicos séricos considerados foram: albumina, creatinina, ureia e ferritina.

\section{Avaliação da ingestão calórica e proteica}

Os dados sobre o consumo alimentar foram obtidos por meio do recordatório alimentar de 24 horas anteriores ao dia da avaliação. A ingestão total de calorias e proteínas ao dia de cada participantes foi calculada por meio de planilha do Microsoft Excel $\circledR$ versão 20I0, contendo a composição centesimal dos alimentos conforme a Tabela Brasileira de Composição de Alimentos ${ }^{(14)}$.

\section{Análise dos dados e aspectos éticos}

Os dados foram tabulados em planilha do Microsoft Excel $\AA$ versão 2010 e analisados no programa estatístico Statistical Package For The Social Sciences (SPSS), versão 23.0, 20 I5. Foram inicialmente apresentados na forma de estatística descritiva (média, desvio-padrão) para caracterizar a amostra estudada.

Para análise das variáveis qualitativas foi utilizado o teste de Qui-quadrado de Pearson, e para as variáveis quantitativas foi utilizado o teste de Mann Whitney. $O$ teste de normalidade Kolmogorov Smirnov foi utilizado para avaliação da distribuição das variáveis.

A análise de regressão logística binária múltipla foi realizada para identificar as principais variáveis relacionadas à presença de sarcopenia. Para se obter um modelo satisfatório, as técnicas estatísticas de seleção das variáveis baseadas em inserção e na retirada das mesmas foram utilizadas (método forward stepwise/backward stepwise). Para melhores ajustes e poder preditivo, a razão de verossimilhança foi utilizada, a significância dos parâmetros da regressão foi analisada pelo teste estatístico de Wald e os testes de Omnibus e Hosmer \& Lemeshow avaliaram a qualidade do modelo. A probabilidade de significância estatística foi considerada com valor de $\mathrm{p}<0,05$.

Esse estudo foi aprovado pelo Comitê de Ética em Pesquisa em Seres Humanos da SES-DF, sob o parecer número 3.135.942 e Certificado de Apresentação para Apreciação Ética número 044956|8.1.0000.5553.

\section{RESULTADOS}

Inicialmente foram recrutados 70 participantes, no entanto, I 8 (25,7I\%) desses não foram elegíveis por estarem nos critérios de exclusão. Nove (12,86\%) participantes tiveram o resultado da avaliação corporal por BIS insatisfatório, outros cinco $(7,14 \%)$ não conseguiram realizar o teste de capacidade funcional e demais quatro $(5,71 \%)$ obedeciam aos critérios de exclusão.A amostra foi constituída por 52 participantes, a média de idade foi $53,90 \pm 14,86$ de anos, com tempo médio de diálise $29,10 \pm 34,80$ de meses.

Os indivíduos com menos de 60 anos eram 32 (6I,54\%) na amostra e os idosos 20 (38,46\%). A maioria dos participantes era do sexo masculino $33(63,46 \%)$, tabela I. Dentre as causas da DRC, a mais prevalente foi a nefropatia diabética $(n=20,38,46 \%)$, nefropatia hipertensiva em 10 (19,23\%), glomerulopatias em quatro (7,69\%) e outras causas em $18(34,62 \%)$ dos participantes.

A presença de desnutrição foi presente em 14 (26,92\%) dos participantes e, pelo instrumento do SARC-F, nove $(17,31 \%)$ apresentaram risco de sarcopenia. A sarcopenia foi presente em I5 (28,85\%) (Tabela I).A sarcopenia severa foi encontrada em sete $(13,46 \%)$ dos indivíduos.

Em relação à presença de sarcopenia, foi observada diferença significativa com a presença de hipertensão $(p=0,007)$. Entre os sarcopênicos $(n=\mid 5,100 \%), 12(80 \%)$ 
eram do sexo masculino, II (73,33\%) hipertensos, 10 $(66,67 \%)$ eram diabéticos, sete $(46,67 \%)$ apresentavam desnutrição por meio da ASG-7p (Tabela I).

Em relação às variáveis quantitativas, os valores observados de IMC, porcentagem de adequação da CMB ângulo de fase e FPP foram, respectivamente, $25,58 \pm 3,89$; $96,7 \mid \pm 11,87 ; 4,89 \pm 1,38$ e $24,33 \pm 10,78$. Sobre a avaliação da ingestão alimentar, o consumo de proteína e calorias dos participantes foi de $I, 0 I \pm 0,30 \mathrm{~g} / \mathrm{kg} / \mathrm{dia}$ e $2 I, 4 I \pm 7,80$ $\mathrm{kcal} / \mathrm{kg} / \mathrm{dia}$, respectivamente (Quadro I).

TABELA 1 - Análise das variáveis qualitativas em relação à presença de sarcopenia dos participantes em diálise peritoneal de uma unidade de nefrologia. Brasília, DF, Brasil, 2019.

\begin{tabular}{|c|c|c|c|c|c|c|}
\hline \multirow{2}{*}{\multicolumn{3}{|c|}{ Variáveis }} & \multicolumn{2}{|c|}{ Sarcopenia } & \multirow{3}{*}{$\begin{array}{c}\text { Total } \\
33 \\
\end{array}$} & \multirow{3}{*}{$\begin{array}{c}\text { p-valor } \\
0,115 \\
\end{array}$} \\
\hline & & & \multirow{2}{*}{$\begin{array}{c}\text { Não } \\
21\end{array}$} & \multirow{2}{*}{$\begin{array}{c}\text { Sim } \\
12\end{array}$} & & \\
\hline Sexo & Masculino & $n$ & & & & \\
\hline & & $\%$ & 56,76 & 80,0 & 63,46 & \\
\hline & Feminino & $\mathrm{n}$ & 16 & 3 & 19 & \\
\hline & & $\%$ & 43,24 & 20,0 & 36,54 & \\
\hline \multirow{4}{*}{ Hipertensão } & \multirow{2}{*}{ Sim } & $\mathrm{n}$ & 37 & 11 & 48 & 0,007 \\
\hline & & $\%$ & 100,00 & 73,33 & 92,30 & \\
\hline & \multirow{2}{*}{ Não } & $\mathrm{n}$ & 0 & 4 & 4 & \\
\hline & & $\%$ & 0,00 & 26,67 & 7,70 & \\
\hline \multirow{4}{*}{ Diabetes } & \multirow{2}{*}{ Sim } & $\mathrm{n}$ & 14 & 10 & 24 & 0,059 \\
\hline & & $\%$ & 37,84 & 66,67 & 46,15 & \\
\hline & \multirow{2}{*}{ Não } & $\mathrm{n}$ & 23 & 5 & 28 & \\
\hline & & $\%$ & 62,16 & 33,33 & 53,85 & \\
\hline \multirow{4}{*}{ Desnutrição $^{\dagger}$} & \multirow{2}{*}{ Sim } & $\mathrm{n}$ & 7 & 7 & 14 & 0,089 \\
\hline & & $\%$ & 18,92 & 46,67 & 26,92 & \\
\hline & \multirow{2}{*}{ Não } & $\mathrm{n}$ & 30 & 8 & 38 & \\
\hline & & $\%$ & 81,08 & 53,33 & 73,08 & \\
\hline \multirow{2}{*}{\multicolumn{2}{|c|}{ Total }} & $\mathrm{n}$ & 37 & 15 & 52 & \\
\hline & & $\%$ & 100,00 & 100,00 & 100,00 & \\
\hline
\end{tabular}

*Teste de Qui-quadrado de Pearson; nível de significância de 5\% $(p<0,05)$.

†Desnutrição: determinada por meio da avaliação subjetiva global de sete pontos.

Fonte: dados da pesquisa.

QUADRO 1 - Variáveis quantitativas dos participantes em diálise peritoneal de uma unidade de nefrologia. Brasília, DF, Brasil, 2019.

\begin{tabular}{|c|c|c|c|}
\hline Parâmetros & $\begin{array}{c}\text { Valor (média e desvio } \\
\text { padrão) }\end{array}$ & Parâmetros & $\begin{array}{c}\text { Valor (média e desvio } \\
\text { padrão) }\end{array}$ \\
\hline Peso seco $(\mathrm{kg})$ & $69,54 \pm 13,50$ & ATM $(\mathrm{kg})$ & $29,30 \pm 12,79$ \\
\hline $\mathrm{CB}(\mathrm{cm})$ & $29,52 \pm 3,66$ & $\mathrm{BCM}(\mathrm{kg})$ & $22,86 \pm 8,54$ \\
\hline $\mathrm{CA}(\mathrm{cm})$ & $96,46 \pm 11,41$ & Resistência $(\Omega)$ & $552,73 \pm 129,49$ \\
\hline $\mathrm{CP}(\mathrm{cm})$ & $34,63 \pm 3,65$ & Reactância $(\Omega)$ & $1480,50 \pm 425,45$ \\
\hline $\mathrm{DCT}(\mathrm{mm})$ & $15,53 \pm 7,29$ & Ângulo de fase & $4,89 \pm 1,38$ \\
\hline IMC (kg/m2) & $25,58 \pm 3,89$ & Força de preensão palmar (kgf) & $24,33 \pm 10,78$ \\
\hline $\mathrm{CMB}(\mathrm{cm})$ & $24,58 \pm 2,80$ & Velocidade de marcha (segundos) & $4,36 \pm 1,22$ \\
\hline Porcentagem de adequação da CMB & $96,71 \pm 11,87$ & Albumina (mg/dL) & $3,79 \pm 0,43$ \\
\hline $\mathrm{OH}(\mathrm{L})$ & $1,96 \pm 3,28$ & Creatinina (mg/dL) & $10,03 \pm 4,91$ \\
\hline TBW (L) & $37,00 \pm 8,45$ & Ureia (mg/dL) & $118,13 \pm 34,41$ \\
\hline ECW (L) & $17,58 \pm 4,48$ & Ferritina $(\mathrm{mg} / \mathrm{dL})$ & $393,75 \pm 339,09$ \\
\hline ICW (L) & $19,42 \pm 4,88$ & Proteína (gramas/dia) & $68,66 \pm 19,18$ \\
\hline E/I & $0,92 \pm 0,19$ & Proteína (gramas/kg de peso/dia) & $1,01 \pm 0,30$ \\
\hline LTI $\left(\mathrm{kg} / \mathrm{m}^{2}\right)$ & $14,76 \pm 4,02$ & Calorias (calorias/dia) & $1438,50 \pm 409,55$ \\
\hline FTI $\left(\mathrm{kg} / \mathrm{m}^{2}\right)$ & $10,80 \pm 4,60$ & Calorias (calorias/kg de peso/dia) & $21,41 \pm 7,80$ \\
\hline LTM (kg) & $40,35 \pm 12,37$ & ASG-7p (pontos) & $6,19 \pm 1,34$ \\
\hline FAT (kg) & $21,54 \pm 9,41$ & SARC-F (pontos) & $1,38 \pm 1,68$ \\
\hline
\end{tabular}

CB: circunferência do braço; CA: circunferência abdominal; CP: circunferência da panturrilha; DCT: dobra cutânea triciptal; IMC: índice de massa corporal; CMB: circunferência muscular do braço; OH: hiper-hidratação; TBW: água total corporal; ECW: água corporal extracelular; ICW: água corporal intracelular; E/I: quociente de água corporal extracelular e água corporal intracelular; LTI: índice de tecido magro; FTI: índice de tecido gordo; LTM: massa de tecido magro; FAT: massa gorda; ATM: massa de gordura corporal; BCM: massa celular corporal; ASG$7 \mathrm{p}$ : avaliação subjetiva global de sete pontos. Fonte: dados da pesquisa. 
$\mathrm{Na}$ análise de associação das variáveis quantitativas em relação à presença de sarcopenia, observou-se diferença significativa para ASG-7p $(p=0,043)$, ângulo de fase $(p=0,005)$ e albumina $(p<0,00 I)$, sendo que os participantes sarcopênicos apresentaram valores significativamente menores que os não sarcopênicos dessas variáveis (Tabela 2).

Inicialmente, as variáveis independentes explicativas definidas, na regressão logística múltipla, para a condição de sarcopenia foram: sexo, idade, tempo de diálise, hipertensão, diabetes, ângulo de fase, albumina, ferritina, ingestão de pro- teína e calorias, ASG-7p e risco cardiovascular, entretanto, nenhuma variável foi estatisticamente significativa (Tabela 3 ).

O método backward stepwise melhorou o modelo de regressão com apenas as variáveis: hipertensão, ângulo de fase e albumina (Tabela 4). Foi observada significância estatística em relação à albumina $(p=0,034)$, sendo o coeficiente da regressão encontrado com valor de -3, I I. Com isso, a relação é inversamente proporcional, a presença da sarcopenia dos participantes está associada aos menores níveis de albumina. Observou-se que uma unidade

TABELA 2 - Associação das variáveis quantitativas em relação à presença de sarcopenia dos participantes em diálise peritoneal de uma unidade de nefrologia. Brasília, DF, Brasil, 2019.

\begin{tabular}{|c|c|c|c|c|c|}
\hline \multicolumn{6}{|c|}{ Sarcopenia } \\
\hline \multirow[t]{2}{*}{ Variável } & \multicolumn{2}{|c|}{ Não } & \multicolumn{2}{|c|}{ Sim } & \multirow[t]{2}{*}{ p-valor } \\
\hline & Mediana & $\begin{array}{l}\text { Amplitude } \\
\text { interquartil }\end{array}$ & Mediana & $\begin{array}{l}\text { Amplitude } \\
\text { interquartil }\end{array}$ & \\
\hline Idade & 56,00 & 23,00 & 55,00 & 20,30 & 0,960 \\
\hline Tempo de diálise & 16,00 & 20,00 & 21,00 & 26,00 & 0,554 \\
\hline ASG-7p ${ }^{\dagger}$ & 7,00 & 0,00 & 5,50 & 3,00 & 0,043 \\
\hline Ângulo de fase & 5,24 & 1,30 & 4,08 & 2,30 & 0,005 \\
\hline Albumina & 3,90 & 0,30 & 3,50 & 0,60 & $<0,001$ \\
\hline Ferritina & 293,00 & 372,50 & 412,10 & 491,70 & 0,237 \\
\hline Proteína gramas $/ \mathrm{kg} / \mathrm{dia}$ & 0,98 & 0,36 & 1,05 & 0,62 & 0,896 \\
\hline Calorias calorias/kg/dia & 19,69 & 9,36 & 19,88 & 10,01 & 0,976 \\
\hline
\end{tabular}

* Teste de Mann Whitney; nível de significância de 5\% $(p<0,05)$.

†ASG-7p: avaliação subjetiva global de sete pontos.

Fonte: dados da pesquisa.

TABELA 3 - Regressão logística para a variável dependente sarcopenia dos participantes em diálise peritoneal de uma unidade de nefrologia. Brasília, DF, Brasil, 2019.

\begin{tabular}{|c|c|c|c|c|}
\hline Variável & Coeficiente & p-valor & $\mathbf{R}^{\dagger}$ & $95 \%$ IC $\ddagger$ \\
\hline Sexo & $-0,98$ & 0,553 & 0,38 & $0,02-9,42$ \\
\hline Idade & $-0,02$ & 0,572 & 0,98 & $0,90-1,06$ \\
\hline Tempo de diálise & $-0,02$ & 0,418 & 0,98 & $0,94-1,03$ \\
\hline Hipertensão & 25,39 & 0,999 & $1,07^{*} 10^{11}$ & $0,00-\S$ \\
\hline Diabetes & 0,74 & 0,691 & 2,09 & $0,05-78,27$ \\
\hline Ângulo de fase & $-1,26$ & 0,131 & 0,28 & $0,05-1,46$ \\
\hline Albumina & $-6,40$ & 0,067 & 0,00 & $0,00-1,58$ \\
\hline Ferritina & 0,00 & 0,925 & 1,00 & $0,99-1,01$ \\
\hline Proteína/kg/dia & 6,97 & 0,134 & 107,94 & $0,12-9662008,62$ \\
\hline Calorias/kg/dia & $-0,21$ & 0,449 & 0,82 & $0,48-1,38$ \\
\hline ASG-7p" & 2,82 & 0,152 & 16,71 & $0,36-79,19$ \\
\hline
\end{tabular}

${ }^{*}$ Nível de significância de $5 \%(p<0,05)$.

†RR: odds ratio/risco relativo.

$\$ 95 \%$ IC: intervalo de confiança de $95 \%$ para risco relativo.

§Valor muito alto não determinado.

IASG-7p: avaliação subjetiva global de sete pontos.

Fonte: dados da pesquisa.

TABELA 4 - Regressão logística com seleção de variáveis para a variável dependente sarcopenia dos participantes em diálise peritoneal de uma unidade de nefrologia. Brasília, DF, Brasil, 2019.

\begin{tabular}{|c|c|c|c|c|}
\hline Variáveis & Coeficiente & p-valor ${ }^{*}$ & $\mathbf{R R}^{\dagger}$ & $95 \% I^{\ddagger}$ \\
\hline Hipertensão & 22,94 & 0,999 & $9,15^{*} 10^{9}$ & $0,00-\S$ \\
\hline Ângulo de fase & $-0,78$ & 0,068 & 0,46 & $0,20-1,06$ \\
\hline
\end{tabular}

${ }^{*}$ Nível de significância de $5 \%(p<0,05)$

†RR: odds ratio/risco relativo.

¥95\%IC: intervalo de confiança de $95 \%$ para risco relativo.

§Valor muito alto não determinado.

Fonte: dados da pesquisa. 
a menos de albumina aumenta as chances da ocorrência de sarcopenia (RR, 22,22; 95\%IC 0,00-0,80; $p=0,034)$.

\section{DISCUSSÃO}

Nesse estudo, observou-se a prevalência de sarcopenia em I5 (28,85\%) participantes, sendo a sarcopenia severa encontrada em sete (13,46\%). A sarcopenia foi prevalente em participantes do sexo masculino $(n=12$, $80,00 \%$ ) e em hipertensos ( $n=11,73,33 \%$ ). Foi encontrada diferença significativa na presença de hipertensão $(p=0,007)$ entre os grupos com e sem sarcopenia.

Abro et al. ${ }^{(15)}$ avaliaram a sarcopenia em 155 participantes, com $63,0 \pm 14,9$ anos de idade, encontrando resultados de $11 \%$ a $15,5 \%$, dependendo da classificação utilizada. Esses autores utilizaram três critérios para definição de sarcopenia: o do Foundation for the National Institutes of Health Sarcopenia Project (FNIH), o do European Working Group on Sarcopenia in Older People (EWGSOP), e do Asian Working Group on Sarcopenia (AWGS), não encontrando diferença significativa entre as recomendações dessas diretrizes. Eles avaliaram a massa muscular por meio de bioimpedância multifrequencial segmentar e sugeriram haver uma prevalência menor de sarcopenia em DP, provavelmente, em virtude das mudanças do estado de hidratação e eletrólitos serem menos frequentes que em hemodiálise. Vale ressaltar que na DP os indivíduos são expostos à absorção de glicose do dialisato o que, potencialmente, pode levar ao ganho de peso, ao aumento no IMC e influência no ajuste do resultado da massa muscular.

Pelos resultados obtidos no presente estudo, não houve associação significativa entre a presença da sarcopenia e as variáveis: idade, presença de diabetes, tempo de diálise, ingestão de proteína e de calorias e níveis séricos de ferritina. Achados semelhantes ao presente estudo foram encontrados por As'habi et al. ${ }^{\left({ }^{(6)}\right)} \mathrm{em}$ um estudo transversal com 79 participantes acima de 18 anos de idade. Esses autores encontraram a condição de sarcopenia em $1 \mathrm{I}, 5 \%$ dos indivíduos, avaliando a massa muscular também por bioimpedância elétrica, sendo a prevalência dessa condição no sexo masculino significativamente maior $(n=8,29 \% ; p=0,009)$. Possivelmente, esse achado deve-se ao fato da redução da secreção de testosterona o que leva à diminuição da síntese e aumento do catabolismo das proteínas musculares nos homens. Também no presente estudo, vale ressaltar, a média $(I, 01 \pm 0,30 \mathrm{gramas} / \mathrm{kg} / \mathrm{dia})$ encontrada para ingestão de proteína dentre os participantes está no limite inferior da recomendação preconizada para DRC em DP(17).

Em relação aos marcadores nutricionais analisados no estudo, a sarcopenia tem uma relação inversa com baixos níveis séricos de albumina, menores valores do ângulo de fase e do escore da ASG-7p, como instrumento para diagnóstico nutricional, sugerindo relação com desnutrição. É bem descrito na literatura a associação existente entre métodos subjetivos e objetivos de avaliação do estado nutricional, sendo o ângulo de fase um bom indicador nutricional e fator de risco independente para mortalidade ${ }^{(18)}$. Vale ressaltar, em ambas situações, tanto sarcopenia e desnutrição, há presença de possíveis mecanismos inflamatórios e de estresse oxidativo envolvidos, principalmente, na vigência da DRC.

A albumina, apesar de não ser um bom marcador nutricional, já que sofre influência, por exemplo, do estado de volemia, pelos resultados obtidos, aumenta em 22,22 vezes as chances de sarcopenia. Em portadores de DRC em DP, pelo processo de diálise utilizando a membrana peritoneal, há maiores perdas de proteínas, o que sugere a importância da manutenção dos níveis de albumina normais para a prevenção da ocorrência de sarcopenia nessa população.

Kamijo et al.(19) em um estudo coorte prospectivo de 18 meses, realizado no Japão, com 119 idosos de $66,8 \pm 3,2$ anos de idade, observaram prevalência de sarcopenia em 10,9\% dos participantes, sendo essa condição relacionada à mortalidade. $A$ avaliação da massa muscular foi realizada por BIS (mesmo instrumento utilizado no presente estudo), contudo, os autores utilizaram o critério do Asian Working Group on Sarcopenia (AWGS) para diagnóstico da sarcopenia. Nesse estudo japonês, nenhuma diferença significativa foi encontrada entre os grupos com e sem sarcopenia em relação às variáveis sexo, diabetes e tempo de diálise, resultado similar ao encontrado na presente pesquisa.

Conforme os resultados encontrados, obteve-se diferença significativa entre os indivíduos com e sem sarcopenia em relação à presença da hipertensão, no entanto, não foi observada relação de casualidade entre essas variáveis por meio da regressão logística. Rosa et al. ${ }^{(20)}$ desenvolveram um estudo transversal para avaliação da sarcopenia e fatores associados em 35 participantes com idade de 54,4 $\pm 18,7$ anos em DPA. Os autores avaliaram a composição corporal por antropometria, encontrando presença de sarcopenia em I4 (4I,2\%) dos participantes, com predomínio no sexo masculino, obtendo parâmetros como sexo, hipertensão, hipertrigliceridemia e indicadores antropométricos de massa muscular associados à presença de sarcopenia.

O presente estudo apresenta algumas limitações. Primeiramente, o delineamento transversal não permitiu visualizar a relação de causa e efeito entre fatores relacionados à sarcopenia e aos desfechos clínicos, como mortalidade, adicionalmente, a amostra reduzida de participantes envolvidos. Outro fator limitante foi a carac- 
terística predominantemente masculina e de indivíduos hipertensos presentes na amostra estudada.

Além disso, vale ressaltar, os pontos de corte para critério da sarcopenia não são bem definidos na literatura, inclusive em relação à população com DRC. Há diversos métodos utilizados para diagnóstico da sarcopenia, no caso para a avaliação da massa muscular, o que, consequentemente, influencia nos resultados de prevalência dessa condição. Mesmo diante dessas limitações, os resultados dessa pesquisa são válidos e concordantes com dados já existentes na literatura.

\section{CONCLUSÃO}

A sarcopenia é prevalente na DRC, especialmente, em homens, e relaciona-se com parâmetros como albumina, ângulo de fase e o escore do método integrativo da avaliação subjetiva global de sete pontos, utilizado para diagnóstico da desnutrição.

A albumina é um fator independente para essa condição e os seus níveis reduzidos aumentam as chances de sarcopenia em indivíduos com DRC em diálise peritoneal, por conseguinte, mais estudos para investigação dos possíveis fatores associados são necessários. 


\section{REFERÊNCIAS}

I. Roth GA,Abate D,Abate KH,Abay SM,Abbafati C,Abbasi N, et al. Global, regional, and national age-sex-specific mortality for 282 causes of death in 195 countries and territories, 1980-2017: a systematic analysis for the Global Burden of Disease Study 2017. The Lancet [Internet]. 2018 [Acesso em 25 de mai 2020]; 392(10159): I736-1788. Disponível em: https://doi.org/10.1016/S0140-6736(18)32203-7.

2. Neves PDMM, Sesso RCC, Thomé FS, Lugon JR, Nascimento MM. Censo Brasileiro de Diálise: análise de dados da década 2009-20 18. J Bras Nefrol [Internet]. 2020 [Acesso em 25 de mai 2020]; 42(2): I91-200. Disponível em: https://doi. org/2175-8239-JBN-2019-0234.

3. Oliveira JF, Marinho CLA, Silva RS, Lira GG. Qualidade de vida de pacientes em diálise peritoneal e seu impacto na dimensão social. Esc Anna Nery [Internet]. 2019 [Acesso em 28 de mai 2020]; 23(I): I-8. Disponível em: http://dx.doi. org/10.1590/2 177-9465-ean-2018-0265.

4. Farias DH, Melo BC, MinatelV, Calles CAN, Lira JLF. Sarcopenia e sua influência na mobilidade de pacientes com doença renal crônica: uma revisão sistemática. ConScientiae Saúde [Internet]. 2019 [Acesso em 25 de mai 2020]; 18(2): 293-300. Disponível em: https://doi.org//0.5585/conssaude.v18n2.10546.

5. Cruz-Jentoft AJ, Sayer AA. Sarcopenia. The Lancet [Internet]. 2019 [Acesso em 25 de mai 2020]; 393:2636-2646. Disponível em: https://doi.org/I0.10 I6/S0 I40-6736(19)31 I38-9.

6. Brasil. Ministério da Saúde. Orientações para coleta e análise de dados antropométricos em serviços de saúde: norma técnica do Sistema de Vigilância Alimentar e Nutricional/ SISVAN. [online]. [Acesso em 7 de jun 2019]. Disponível em: https://bvsms.saude.gov.br/bvs/publicacoes/orientacoes_coleta_analise_dados_antropometricos.pdf. Ind ed. Brasília: Ministério da Saúde; 201 I.

7. Frisancho AR. New norms of upper limb at and muscle áreas for assessment of nutritional status. Am J Clin Nutr [Internet]. 198I [Acesso em 7 de jun 2019]; 34(I I): 2540-2545. Disponível em: https://doi.org/10.1093/ajcn/34.I I.2540.

8. Lim SL, Lin XH, Daniels L. Seven-Point Subjective Global Assessment Is More Time Sensitive Than Conventional Subjective Global Assessment Detecting Nutrition Changes. J Parenter Enteral Nut [Internet]. 2015 [Acesso em 7 de jun 2019]; 40(7): 966-972. Disponível em: https://doi. org/I0.I I77/0 | 48607| I 5579938.

9. Cruz-Jentoft AJ, Bahat G, Bauer J, Boirie Y, Bruyère Y, Cederholm T, et al. Sarcopenia: revised European consensus on definition and diagnosis. Age Aging [Internet]. 2019 [Acesso em 7 de jun 2019]; 48: 16-3I. Disponível em: https://doi.org//0.1093/ageing/afyl69.

10. Malmstrom TK, Miller DK, Simonsick EM et al. SARC-F: a symptom score to predict persons with sarcopenia at risk for poor functional outcomes. J Cachexia Sarcopenia Muscle [Internet]. 2016 [Acesso em 7 de jun 2019]; 7: 28-36. Disponível em: https://doi.org/I0.1002/jcsm. 12048.
I I. National Health and Nutrition Examination Survey. [online]. [Acesso em 21 de jan 2020]. Disponível em https://www. cdc.gov/nchs/nhanes/index.htm.

12. Massy-Westropp, Rankin W, Ahern M, Krishnan J, Hearn TC. Measuring grip sthrength in normal adults: reference ranges and a comparison of electronic and hydraulic instruments. The J Hand Surgery [Internet]. 2004 [Acesso em 7 de jun 2019]; 29A(3): 5 |4-5 I9. Disponível em: https://doi. org/10.1016/j.jhsa.2004.01.012.

13. Gonzalez MC, Barbosa-Silva TG, Heymsfield SB. Bioelectrical impedance analysis in the assessment of sarcopenia. Curr Opin Clin Nutr Metab Care [Internet]. 20 I8; [Acesso em 25 de mai 2020]; 2 I (5): 366-74. Disponível em: https:// doi: 10.1097/MCO.0000000000000496.

14. NEPA. Tabela Brasileira de Composição de Alimentos. [online]. [Acesso em 21 de jan 2020]. Disponível em: http://www.nepa. unicamp.br/taco/index.php. 4nd ed. São Paulo:UNICAMP;20I I.

15.Abro A, Delicata LA, Vongsanim S, Davenport A. Differences in the prevalence of sacopenia in peritneal dialysis patients using hand grip strength and apendicular lean mass: depends upon guideline definitions. Eur J Clin Nutr [Internet]. 2018 [Acesso em 25 de mai 2020]; 72(7): 993-9. Disponível em: https://doi: 10.1038/s4|430-018-0238-3.

I6.As'habi A, Najafi I,Tabibi H, Hedayati M. Prevalence of sarcopenia and dynapenia and their determinants in iranian peritoneal dialysis patients. Iran J Kidney Dis [Internet]. 2018 [Acesso em 25 de mai 2020]; I2(I): 53-60. Disponível em: http://www.ijkd.org/index.php/ijkd/article/view/3493/985.

17. Ikizler TA, Burrowes JD, Byham-Gray LD, Campbell KL, Carrero JJ, Chan W, et al. KDOQI Clinical practice guidelines for nutrition in CKD: 2020 UPDATE. Am J Kidney Dis [Internet]. 2020 [Acesso em 30 de ago 2020]; 76(3): SI-SI08. Disponível em: https://doi.org/I0.1053/j.ajkd.2020.05.006.

18. Duarte RS, Pinho CPS, Barboza YACO, Silva CMCS, CarvaIho TR, Lemos MCC.Asociación del ángulo de fase com parâmetros de evaluación del estado nutricional em pacientes em hemodiálisis. Rev Chil Nut [Internet]. 2019 [Acesso em 25 de mai 2020]; 46(2): 99-106. Disponível em: http:// dx.doi.org//0.4067/s07| 7-75 I82019000200099.

19. Kamijo Y, Kanda E, Ishibashi Y, Yoshida M. Sarcopenia and frailty in PD: impact on mortality, malnutrition, and inflammation. Perit Dial Int [Internet]. 2018 [Acesso em 25 de mai 2020]; 38(6): 447-54. Disponível em: https://doi.org/l0.3747\%2Fpdi.2017.0027l.

20. Rosa NCP, Alcântara FG, Cattafesta M, Furriel AF, Salaroli LB. Sarcopenia e fatores associados em pacientes com doença renal crônica em tratamento dialítico peritoneal. Saúde e pesquisa [Internet]. 2019 [Acesso em 25 de mai 2020]; II(3): 483-93. Disponível em: https://doi.org//0.17765/ 2I76-9206.20I8v I In3p483-493.

Recebido: 2020-09-20

Aceito: 2020-10-23 